\title{
Conserved quantities in general relativity and quantum anomalies
}

\author{
B.P. Dolan* \\ Dept. of Theoretical Physics, Maynooth University, Maynooth, Ireland \\ School of Theoretical Physics, Dublin Institute for Advanced Studies, 10 Burlington Rd., \\ Dublin 8, Ireland \\ E-mail: bdolanethphys.nuim.ie
}

\begin{abstract}
Wald's construction of the Noether charge associated with a Killing vector of a solution of a diffeomorphism invariant theory is reviewed. A variation of a solution of the equations of motion is a 1-form on the space of solutions and a symplectic form on the space of solutions is a 2-form . The cohomological nature of the construction is emphasised and this allows conserved quantities to be evaluated without having to go to asymptotic infinity.

The construction gives rise to a double differential complex associated with the exterior derivative on space-time and the exterior derivative on the space of solutions. It is shown that the same double differential complex arises in the discussion of quantum anomalies in gauge theories and gravity.
\end{abstract}

DIAS-STP-2004

Corfu Summer Institute 2019 "School and Workshops on Elementary Particle Physics and Gravity" (CORFU2019)

31 August - 25 September 2019

Corfu, Greece

${ }^{*}$ Speaker. 


\section{Introduction}

The connection between symmetries and conserved quantities discovered by Emmy Noether in 1918 [1] had profound impact on the development of physics in the twentieth century. Conservation laws are related to symmetries and phase space and in 1987 Crnković and Witten gave a general construction for a symplectic structure on the space of solutions of general relativity [2]. This was generalised by Wald and Lee to a general diffeomorphic invariant field theory $[3,4]$. In this talk we re-formulate Lee and Wald's formulation in the language of differential forms on the infinite dimensional space of solutions of the equations of motion. This leads to a double differential complex involving differential forms and exterior derivatives on space-time entwined with differential forms and exterior derivatives on the space of solutions of the equations of motion. We show that the same differential complex arises in the discussions of quantum anomalies in gauge theories and general relativity $[5,6,7]$.

\section{Noether's theorem}

We first give a quick review of Noether's Theorem for internal in differential form language before going on to consider diffeomorphism invariance. For internal symmetries Noether's theorem is quickly obtained from Stokes' theorem. Consider a system in $D+1$ dimensions with dynamical fields $F^{I}$, which may or may not include a metric, governed by a Lagrangian $L\left(F^{I}, \partial_{\mu} F\right)$ which is a $(D+1)$-form on space-time. Under an arbitrary variation of the fields

$$
\delta L\left(F^{I}\right)=E_{J}\left(F^{I}\right) \delta F^{J}+d \boldsymbol{\theta}\left(F^{I}, \delta F^{I}\right)
$$

where $E_{I}\left(F^{I}\right)=0$ are the equations of motion.

If $\mathscr{T}$ generates a symmetry of $L$, then under a variation $\delta_{\mathscr{T}}=\varepsilon \mathscr{T}, \delta_{\mathscr{T}} L=0$ for any field configuration $F^{I}$. For a configuration $S^{I}$ which is a solution of the equations of motion,

$$
d \boldsymbol{\theta}\left(S^{I}, \boldsymbol{\delta}_{\mathscr{T}} S^{I}\right)=0
$$

on shell. We shall denote the set of all field configurations that satisfy the equations of motion by $\mathscr{S}$, so $S^{I} \in \mathscr{S}$.

Thus $\boldsymbol{\theta}\left(S^{I}, \boldsymbol{\delta}_{\mathscr{T}} S^{I}\right)$ is exact and, integrating over space-time $\mathscr{M}$

$$
\int_{\mathscr{M}} d \boldsymbol{\theta}=0
$$

Assuming that the fields fall off fast enough at space-like infinity (the time-like tube $\boldsymbol{T}$ in the figure below) $\mathscr{M}$ is effectively bounded by hypersurfaces $\Sigma$ and $\Sigma^{\prime}$. 


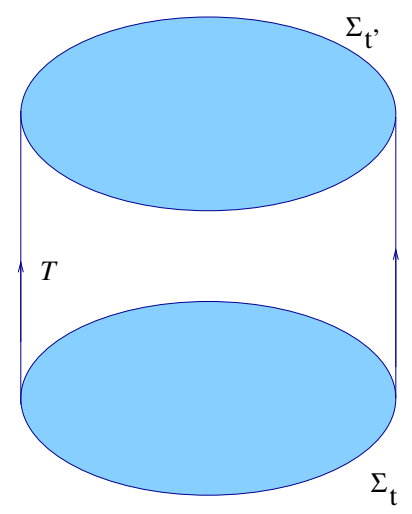

We then have

$$
\int_{\mathscr{M}} d \boldsymbol{\theta}=\int_{\Sigma} \boldsymbol{\theta}-\int_{\Sigma^{\prime}} \boldsymbol{\theta}=0
$$

With $\boldsymbol{\theta}=* \boldsymbol{j}\left(\theta_{\mu v \rho}=\varepsilon_{\mu \nu \rho \sigma} j^{\sigma}\right)$ the Noether charge $\mathscr{Q}$ is now defined as

$$
\mathscr{Q}=\int_{\Sigma} * \boldsymbol{j}=\int_{\Sigma^{\prime}} * \boldsymbol{j} \quad\left(=\int_{\Sigma} j_{\mu} d \Sigma^{\mu}\right)
$$

\section{Phase space formulation}

The $D$-form $\boldsymbol{\theta}$ is also central to constructing the phase space formulation of a field theory. Under a second variation define (again on-shell $S^{I} \in \mathscr{S}$ ) define a

$$
\boldsymbol{\omega}\left(S^{I}, \delta_{1} S^{I}, \delta_{2} S^{I}\right):=\delta_{1} \boldsymbol{\theta}_{2}-\delta_{2} \boldsymbol{\theta}_{1}
$$

where $\boldsymbol{\theta}_{1}=\boldsymbol{\theta}\left(S^{I}, \delta_{1} S^{I}\right)$ and $\boldsymbol{\theta}_{2}=\boldsymbol{\theta}\left(S^{I}, \delta_{2} S^{I}\right)$. We can now define a symplectic form on the phasespace of solutions $\boldsymbol{\Omega}$ via

$$
\Theta=\int_{\Sigma} \boldsymbol{\theta}, \quad \boldsymbol{\Omega}=\boldsymbol{\delta} \Theta=\int_{\Sigma} \boldsymbol{\omega}
$$

where $\boldsymbol{\delta}$ is the exterior derivative on the (infinite dimensional) space of solutions, $\boldsymbol{\delta}^{2}=0$. As we shall see if there are gauge symmetries $\boldsymbol{\Omega}$ is a pre-symplectic from, in the sense defined below, rather than a true symplectic form.

There are now two exterior derivatives, $d$ acting on differential forms on finite-dimensional space-time and $\boldsymbol{\delta}$ acting on differential forms on the infinite dimensional space of solutions of the equations of motion. These commute $d \boldsymbol{\delta}=\boldsymbol{\delta} d$.

The pre-symplectic form $\boldsymbol{\Omega}$ is unaffected if the Lagrangian is changed by adding a total derivative,

$$
\boldsymbol{L} \rightarrow \boldsymbol{L}+d \boldsymbol{\alpha} \Rightarrow \boldsymbol{\theta} \rightarrow \boldsymbol{\theta}+\boldsymbol{\delta} \boldsymbol{\alpha}
$$

and $\boldsymbol{\Omega}=\boldsymbol{\delta} \boldsymbol{\Theta}$ is invariant since $\boldsymbol{\delta}^{2}=0$.

The simplest case is that of a free scalar field $\phi$ with Lagrangian density

$$
\boldsymbol{L}=-\frac{1}{2} d \phi \wedge * d \phi-m^{2} \phi^{2} * 1 .
$$


In this case $\boldsymbol{\theta}=-(\boldsymbol{\delta} \phi) \wedge * d \phi$ and the conjugate momentum is $\pi=\dot{\phi}$ giving

$$
\boldsymbol{\omega}=(\boldsymbol{\delta} \phi) \wedge * d(\boldsymbol{\delta} \phi) .
$$

Choosing a $t=$ const hypersurface $\Sigma$,

$$
\boldsymbol{\Omega}=\int_{\Sigma}(\boldsymbol{\delta} \phi) \wedge * \boldsymbol{\delta}(d \phi)=\int_{\Sigma}(\boldsymbol{\delta} \phi) \wedge(\boldsymbol{\delta} \pi) \tilde{*} 1
$$

where $\tilde{*} 1$ is the volume form on the $D$-dimensional hypersurface $\Sigma$. The symplectic structure here is of exactly the form one would expect from Darboux's theorem.

In general the variation $\boldsymbol{\delta} S^{I}$ on the space of solutions $\mathscr{S}$ can include gauge transformations and diffeomorphisms, $\mathscr{G}$. When this is the case we must project $\boldsymbol{\Omega}$ from the space of solutions $\mathscr{S}$ to the space of solutions modulo gauge transformation and/or diffeomorphisms, $\widehat{\mathscr{S}}=\mathscr{S} / \mathscr{G}$. There must then be a symplectic 2-form $\widehat{\boldsymbol{\Omega}}$ on $T^{*} \widehat{\mathscr{S}}$ which pulls-back to $\boldsymbol{\Omega}$ on $T^{*} \mathscr{S}$ under this projection. Under these circumstances we expect that $\boldsymbol{\Omega}$ is degenerate and $\boldsymbol{\Omega}=\int_{\Sigma} \boldsymbol{\omega}$ must vanish if one of the field variations is a diffeomorphism. Diffeomorphisms generated by a vector field $\vec{X}$ are represented by Lie derivatives $\mathscr{L}_{\vec{X}}$ and it is convenient to introduce a constant 1-form $\varepsilon$ on $T^{*} \mathscr{S}$ which anti-commutes with $\boldsymbol{\delta}, \boldsymbol{\varepsilon} \boldsymbol{\delta}=-\boldsymbol{\delta} \boldsymbol{\varepsilon}$ and commutes with $d, d \boldsymbol{\varepsilon}=\boldsymbol{\varepsilon} d$. With this notation a variation corresponding to a diffeomorphism is written as ${ }^{1}$

$$
\boldsymbol{\delta}_{\vec{X}} S^{I}=\boldsymbol{\varepsilon} \mathscr{L}_{\vec{X}} S^{I}=\boldsymbol{\varepsilon} d i_{\vec{X}} S^{I}
$$

We expect that $\boldsymbol{\Omega}$ will vanish if one of the variations in (3.1) is a diffeomorphism and this will be the case if

$$
\boldsymbol{\omega}=d(\boldsymbol{\varepsilon} \wedge \boldsymbol{\eta}(\vec{X}))
$$

for some field dependent $(D-1)$-form space-time $\boldsymbol{\eta}(\vec{X})$ (which is a 1 -form on $\mathscr{T}^{*} \mathscr{S}$ ). Provided field variations vanish on $\partial \Sigma$, the boundary of $\Sigma$, we will have [2,3]

$$
\boldsymbol{\Omega}=\int_{\Sigma} \boldsymbol{\omega}=\boldsymbol{\varepsilon} \wedge \int_{\Sigma} d \boldsymbol{\eta}(\vec{X})=\boldsymbol{\varepsilon} \wedge \int_{\partial \Sigma} \boldsymbol{\eta}(\vec{X})=0 .
$$

In particular if a Hamilton $h(\vec{X})$ exists that generates the flow $\vec{X}$ on phase space then

$$
d \boldsymbol{\eta}(\vec{X})=\boldsymbol{\delta} h(\vec{X})
$$

and

$$
\boldsymbol{\omega}=-\boldsymbol{\delta}(\boldsymbol{\varepsilon} h(\vec{X}))=d(\boldsymbol{\varepsilon} \wedge \boldsymbol{\eta}(\vec{X})) \quad \Rightarrow \quad \boldsymbol{\Omega}=0
$$

when one of the variations is a diffeomorphism vanishing at spatial infinity.

When such a hamiltonian exists we can define

$$
\mathscr{H}[\vec{X}]:=\int_{\Sigma} h(\vec{X})
$$

and

$$
\boldsymbol{\delta} \mathscr{H}[\vec{X}]=\int_{\Sigma} \boldsymbol{\delta} h(\vec{X})=\int_{\partial \Sigma} \boldsymbol{\eta}(\vec{X})=0
$$

if $\vec{X}$ vanishes on $\partial \Sigma$. For example in general relativity, for a time-like vector field $\vec{X}, \mathscr{H}[\vec{X}]=0$ is a constraint.

\footnotetext{
${ }^{1} i_{\vec{X}}$ denotes contraction of a differential from with the vector $\vec{X}$ and $\mathscr{L}_{\vec{X}}=d i_{\vec{X}}+i_{\vec{X}} d$ on forms.
} 


\section{Killing symmetries}

If $\vec{X}=\vec{K}$ is a Killing vector then $\mathscr{L}_{\vec{K}} S^{I}$ either vanishes or is a gauge transformation and we assume the symplectic density is invariant, $\boldsymbol{\omega}=0$, in which case $\boldsymbol{\eta}$ is $d$-closed

$$
d \boldsymbol{\eta}(\vec{K})=0 .
$$

However $\vec{K}$ need not vanish on $\partial \Sigma$ and this can lead to non-trivial conserved quantities.. If $\Sigma$ has an inner and an outer boundary, $\partial \Sigma=\sigma_{1} \cup \sigma_{2}$

then

$$
\int_{\Sigma} d \boldsymbol{\eta}(\vec{K})=\int_{\sigma_{2}} \boldsymbol{\eta}(\vec{K})-\int_{\sigma_{1}} \boldsymbol{\eta}(\vec{K})=0
$$

and $\int_{\sigma} \boldsymbol{\eta}(\vec{K})$ is independent of the surface $\sigma$. Furthermore if $\boldsymbol{\varepsilon} \wedge \boldsymbol{\eta}(\vec{K})=\boldsymbol{\delta} \boldsymbol{\psi}(\vec{K})$ is $\boldsymbol{\delta}$-exact for some $\boldsymbol{\psi}$ (which might not be the case — this depends crucially on the specific Lagrangian) then an invariant can be constructed [4]. Let

$$
\boldsymbol{\psi}(\vec{K})=\boldsymbol{\varepsilon} * \mathscr{J}(\vec{K})
$$

where $\mathscr{J}$ is a 2 -form on space-time - the Noether 2-form. Then

$$
\mathscr{Q}(\vec{K})=\int_{\sigma} * \mathscr{J}
$$

is independent of the $(D-1)$-dimensional surface $\sigma$ on which it is evaluated. Of course $\mathscr{Q}(\vec{K})$ does depend on the solution, changing the solution of the equations of motion does change $\mathscr{Q}$ in general and $\int_{\sigma} \boldsymbol{\eta}(\vec{K})=\boldsymbol{\delta} \mathscr{Q}[\vec{K}]$.

To summarise we have a (pre)-symplectic form $\boldsymbol{\Omega}=\int_{\Sigma} \boldsymbol{\omega}$. which is a 2 -form on $T^{*} \mathscr{S}$. If one of the field variations is a diffeomorphism generated by a vector field $\vec{X}$ which vanishes on the boundary of $\Sigma$ then

$$
\boldsymbol{\omega}=\boldsymbol{\varepsilon} \wedge d \boldsymbol{\eta}(\vec{X}) .
$$

For a Killing vector $\boldsymbol{\omega}=0$ and $\boldsymbol{\eta}(\vec{K})$ is $d$-closed but it is not necessarily the case that $\vec{K} \neq 0$ on $\partial \Sigma$. If in addition there exists a 2 -from $\mathscr{J}$ on space-time such that $\boldsymbol{\eta}$ is $\boldsymbol{\delta}$-exact, $\boldsymbol{\eta}=-\boldsymbol{\delta} * \mathscr{J}$, then

$$
\mathscr{Q}[\vec{K}]=\int_{\sigma} * \mathscr{J}
$$

is an invariant associated with the Killing vector $\vec{K}$.

\subsection{Example: Schwarzschild black-hole mass in General Relativity}

Perhaps the simplest example exhibiting diffeomorphism invariance is that of a Schwarzschild black-hole in General Relativity. The Einstein Lagrangian (with $G=c^{2}=1$ ) is

$$
L=\frac{1}{16 \pi} R_{a b} \wedge * e^{a b}
$$

where $e^{a}$ are orthonormal 1-forms, $e^{a b}=e^{a} \wedge e^{b}$ and the curvature 2-forms are

$$
R_{a b}=d \omega_{a b}+\omega_{a c} \wedge \omega_{b}^{c}
$$


for torsion-free connection 1-forms $\omega_{a b}$ satisfying

$$
d e^{a}+\omega_{b}^{a} \wedge e^{b}=0
$$

Under a field variation on finds [8]

$$
\boldsymbol{\theta}=\left(\boldsymbol{\delta} \omega_{a b}\right) \wedge * e^{a b}
$$

and

$$
\boldsymbol{\eta}(\vec{K})=\frac{1}{16 \pi}\left(i_{\vec{K}} \boldsymbol{\theta}+\boldsymbol{\delta} * d K\right) .
$$

In particular the Schwarzschild metric

$$
d s^{2}=-\left(1-\frac{2 m}{r}\right) d t^{2}+\frac{d r^{2}}{\left(1-\frac{2 m}{r}\right)}+r^{2}\left(d \vartheta^{2}+\sin ^{2} \vartheta d \varphi^{2}\right)
$$

has a time-like Killing vector $\vec{K}=\frac{\partial}{\partial t}$ and under the metric variation $m \rightarrow m+\boldsymbol{\delta} m$ one finds

$$
\boldsymbol{\eta}(\vec{K})=\frac{1}{4 \pi} \frac{\boldsymbol{\delta} m}{r^{2}} \boldsymbol{e}^{23} \Rightarrow \mathscr{Q}[\vec{K}]=\frac{1}{4 \pi} \int_{S^{2}} \frac{m}{r^{2}} \boldsymbol{e}^{23}=m
$$

where $e^{2}=r d \vartheta, e^{3}=r \sin \vartheta d \varphi$ and $S^{2}$ is a sphere of constant radius surrounding the origin. Note that any finite $r$ will do, as long as it is outside the event horizon so that the $S^{2}$ is space-like. It is not necessary to take $r \rightarrow \infty$ to calculate the mass. It was shown in [9] that this procedure gives the ADM mass [10] for a general stationary asymptotically flat black hole, provided the $S^{2}$ lies completely outside the mass distribution. It was shown in [11] that it also reproduces the BrownYork mass [12] (which is a priori defined as a difference of two solutions so can be viewed as a 1 -from $\boldsymbol{\delta} m$ on the space of solutions) and the Bondi mass [13] when $S^{2}$ is at a light-like separation from the mass distribution.

\section{Double differential complex}

In Wald's construction defined above there is a possible obstruction to defining $\mathscr{Q}[\vec{K}]$ if

$$
\boldsymbol{\varepsilon} \wedge \boldsymbol{\eta}(\vec{K}) \neq \boldsymbol{\delta} \boldsymbol{\psi}(\vec{K})
$$

and this leads us into cohomology.

Let $W^{p, q}$ be the space of $p$-forms on $T^{*} \mathscr{M}$ and $q$-forms on $T^{*} \mathscr{S}$ and let

$$
W^{r}=\bigoplus_{p+q=r} W^{p, q}
$$

be the space of forms of total degree $r$. Then the differential operator

$$
\boldsymbol{D}=\boldsymbol{\delta}+(-1)^{p} d
$$

maps $W^{r}$ to $W^{r+1}$ with $\boldsymbol{D}^{2}=0$. We then have the double differential complex shown below [8] 


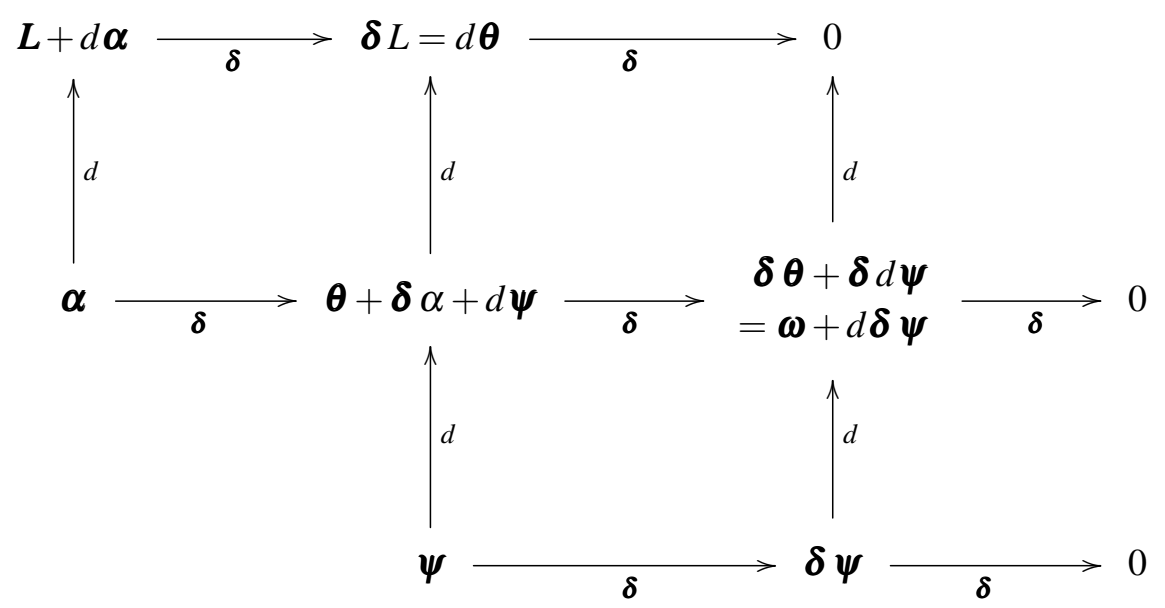

a mathematical structure which is very powerful in cohomology analysis [14].

\section{Anomalies}

The constraint $\boldsymbol{\delta} \boldsymbol{L}=d \boldsymbol{\theta}$ is very reminiscent of the Stora-Zumino descent equations for quantum anomalies in gauge theories $[5,6,7]$. The double differential complex structure is the same for both classically conserved quantities and quantum anomalies.

Wald constructed a generalised Noether charge associated with any diffeomorphic invariant field theory which is based on differential forms on the phase space of solutions. Depending on the field theory there may be cohomological obstructions to defining conserved charges and the mathematical framework is identical to that of the Stora-Zumino consistency conditions and their role in the study of quantum anomalies.

\section{References}

[1] E. Noether, "Invariante Variationsprobleme" [Invariant Variation Problems]. Nachr. D. KÃúnig. Gesellsch. D. Wiss. (in German) 918 (1918) 235. [Translated by M.A. Tavel arXiv:physics/0503066].

[2] C. Crnković and E. Witten, Covariant description of canonical formalism in geometrical theories in Three Hundred Years of Gravitation, eds W. Israel and S.W. Hawking, (1987) CUP.

[3] J. Lee and R.M. Wald, J. Math. Phys. 31 (1990) 725.

[4] R.M. Wald, Phys. Rev. D 48 (1993) 3427, [arXiv:gr-qc/9307038].

[5] A. Bilal, Lectures on Anomalies, [arXiv:hep-th/0802.0634].

[6] L. Alvarez-Gaumé and E. Witten, Nucl. Phys. B 234 (1984) 269.

[7] L. Alvarez-Gaumé and P.H. Ginsparg, Annals Phys. 161 (1985) 423.

[8] B.P. Dolan, Phys. Rev. D 98, 044009 (2018), [arXiv:1804.07689].

[9] V. Iyer and R.M. Wald, Phys. Rev. D 50, 846 (1994), [arXiv:gr-qc/9403028].

[10] R. Arnowitt, S. Deser and C.W. Misner, Phys. Rev. 117, 1595 (1960).

[11] B.P. Dolan, Phys. Rev. D 98, 044010 (2018), [arXiv:1804.10451]. 
[12] J.D. Brown and J.W. York, Jr, Phys. Rev. D 47, 1407 (1993).

[13] H. Bondi, Nature 186, 535 (1960).

[14] R. Bott and L.W. Tu, Differential forms in algebraic topology (Graduate Texts in Mathematics: 82, Springer (1982). 